\title{
Norm approximation for many-body quantum dynamics and Bogoliubov theory
}

\author{
Phan Thành Nam and Marcin Napiórkowski
}

\begin{abstract}
We review some recent results on the norm approximation to the Schrödinger dynamics. We consider $N$ bosons in $\mathbb{R}^{3}$ with an interaction potential of the form $N^{3 \beta-1} w\left(N^{\beta}(x-y)\right)$ with $0 \leq \beta<1 / 2$, and show that in the large $N$ limit, the fluctuations around the condensate can be effectively described using Bogoliubov approximation.
\end{abstract}

\section{Introduction}

In 1924-25, Bose [11] and Einstein [15] predicted that at a very low temperature, many bosons condense into a common quantum state. It took 70 years until this phenomenon was first observed by Cornell, Wieman and Ketterle [4, 13]. Since then, many interesting questions remain unsolved from the theoretical point of view. In fact, Bose and Einstein only considered the ideal gas. The study of interacting Bose gas was initiated in 1947 by Bogoliubov [10]. Roughly speaking, Bogoliubov theory is based on the reduction to quasi-free states, which can be seen as the bosonic analogue to the Bardeen-Cooper-Schrieffer theory [6] for superconductivity.

In the last decades, there have been many attempts to justify Bogoliubov theory from the first principles of quantum mechanics, namely from Schrödinger equation. In the context of the ground state problem, this has been done successfully for one and two-component Bose gases [35, 36, 49], for the Lee-Huang-Yang formula

Phan Thành Nam

Department of Mathematics and Statistics, Masaryk University, Kotlářská 2, 61137 Brno, Czech Republic, e-mail: ptnam@math.muni.cz

Marcin Napiórkowski

Department of Mathematical Methods in Physics, Faculty of Physics, University of Warsaw, Pasteura 5, 02-093 Warszawa, Poland, e-mail: marcin.napiorkowski@fuw.edu.pl

A contribution for the volume "Advances in Quantum Mechanics: contemporary trends and open problems" of the INdAM-Springer series 
of homogeneous, dilute gases $[19,28,50]$ and for the excitation spectrum in the mean-field regime $[47,23,32,14,44]$. In the context of the dynamical problem, Bogoliubov theory has been used widely to study the quantum dynamics of coherent states in Fock space [29, 21, 22, 46, 26, 27, 24, 30, 9, 25]. Very recently, Lewin, Schlein and one of us [31] were able to justify Bogoliubov theory as a norm approximation for the $N$-particle quantum dynamics in the mean-field regime. In [40, 41], we revisited the approach in [31] and extended it to the case of a dilute gas. In the following, we will review our results in $[40,41]$ and explain the ideas of the proof.

We consider a system of $N$ bosons in $\mathbb{R}^{3}$, described by a wave function $\Psi_{N}(t)$ in the Hilbert space $\mathfrak{H}^{N}=\bigotimes_{\text {sym }}^{N} L^{2}\left(\mathbb{R}^{3}\right)$. The system is governed by Schrödinger equation $\Psi_{N}(t)=e^{-i t H_{N}} \Psi_{N}(0)$ with a typical $N$-body Hamiltonian

$$
H_{N}=\sum_{j=1}^{N}-\Delta_{x_{j}}+\frac{1}{N-1} \sum_{1 \leq j<k \leq N} w_{N}\left(x_{j}-x_{k}\right)
$$

We are interested in the delta-type interaction

$$
w_{N}(x-y)=N^{3 \beta} w\left(N^{\beta}(x-y)\right)
$$

where $w \geq 0$ is a fixed function which is nice enough (smooth, compact support, radially symmetric and decreasing). We put the coupling constant $1 /(N-1)$ in order to make the kinetic energy and interaction energy comparable in the large $N$ limit.

The parameter $\beta \geq 0$ describes the character of the interaction between the particles. In the mean-field regime $\beta<1 / 3$, there are many but weak collisions and it is naturally to treat the particles as if they were independent but subjected to a common self-consistent mean-field potential. In the dilute regime $\beta>1 / 3$, there are few but strong collisions and the particles are more correlated. The latter regime is more relevant physically, but also more difficult mathematically.

Our motivation is that $\Psi_{N}(0)$ is the ground state of a trapped system and the time evolution $\Psi_{N}(t)$ is observed when the trapping potential is turned off. From the rigorous result on the ground state in [32], we will assume that

$$
\Psi_{N}(0)=\sum_{n=0}^{N} u(0)^{\otimes(N-n)} \otimes_{s} \varphi_{n}(0)=\sum_{n=0}^{N} \frac{\left(a^{*}(u(0))\right)^{N-n}}{\sqrt{(N-n) !}} \varphi_{n}(0)
$$

where $u(0)$ is a normalized function in $L^{2}\left(\mathbb{R}^{3}\right)$ which describes the condensate and $\Phi(0)=\left(\varphi_{n}(0)\right)_{n=0}^{\infty}$ is a state in the Fock space $\mathscr{F}\left(\left\{u_{0}\right\}^{\perp}\right)$ (see (4) below) for excited particles. Here we use the usual notations of the annihilation and creation operators

$$
a^{*}(f)=\int_{\mathbb{R}^{3}} f(x) a_{x}^{*} \mathrm{~d} x, \quad a(f)=\int_{\mathbb{R}^{3}} \overline{f(x)} a_{x} \mathrm{~d} x, \quad \forall f \in \mathfrak{H},
$$

which satisfy $\left[a_{x}^{*}, a_{y}^{*}\right]=\left[a_{x}, a_{y}\right]=0,\left[a_{x}, a_{y}^{*}\right]=\delta(x-y)$.

When $\beta=0$, it was shown in [31] that if (1) holds then 


$$
\lim _{N \rightarrow \infty}\left\|\Psi_{N}(t)-\sum_{n=0}^{N} u(t)^{\otimes(N-n)} \otimes_{s} \varphi_{n}(t)\right\|=0
$$

(see also the recent work [39] for another approach). Here $u(t)$ is the evolution of the condensate, governed by Hartree equation

$$
i \partial_{t} u(t)=\left(-\Delta+w_{N} *|u(t)|^{2}-\mu_{N}(t)\right) u(t), \quad u(t=0)=u(0)
$$

with the phase parameter $\mu_{N}(t)$ which can be chosen as

$$
\mu_{N}(t)=\frac{1}{2} \iint_{\mathbb{R}^{3} \times \mathbb{R}^{3}}|u(t, x)|^{2} w_{N}(x-y)|u(t, y)|^{2} \mathrm{~d} x \mathrm{~d} y .
$$

The vector $\Phi(t)=\left(\varphi_{n}(t)\right)_{n=0}^{\infty}$ in (2) is a state in the excited Fock space

$$
\mathscr{F}_{+}(t)=\mathscr{F}\left(\{u(t)\}^{\perp}\right)=\bigoplus_{n=0}^{\infty} \mathfrak{H}_{+}(t)^{n}, \quad \mathfrak{H}_{+}(t)^{n}=\bigotimes_{\text {sym }}^{n}\{u(t)\}^{\perp}
$$

and its evolution is determined by Bogoliubov equation

$$
i \partial_{t} \Phi(t)=\mathbb{H}(t) \Phi(t), \quad \Phi(t=0)=\Phi(0) .
$$

Here $\mathbb{H}(t)$ is a quadraric Hamiltonian in Fock space:

$$
\mathbb{H}(t)=\mathrm{d} \Gamma(h(t))+\frac{1}{2} \iint_{\mathbb{R}^{3} \times \mathbb{R}^{3}}\left(K_{2}(t, x, y) a_{x}^{*} a_{y}^{*}+\overline{K_{2}(t, x, y)} a_{x} a_{y}\right) \mathrm{d} x \mathrm{~d} y,
$$

which is obtained from Bogoliubov approximation (which we will explain in Section 2). We use the notations $\mathrm{d} \Gamma(A)=\int a_{x}^{*} A_{x} a_{x} \mathrm{~d} x$ (for example, $\mathrm{d} \Gamma(1)=\mathscr{N}$ is the number operator) and

$$
\begin{aligned}
h(t) & =-\Delta+|u(t, \cdot)|^{2} * w_{N}-\mu_{N}(t)+Q(t) \widetilde{K}_{1}(t) Q(t), \\
K_{2}(t) & =Q(t) \otimes Q(t) \widetilde{K}_{2}(t), \quad Q(t)=1-|u(t)\rangle\langle u(t)|,
\end{aligned}
$$

where $\widetilde{K}_{2}(t, x, y)=u(t, x) w_{N}(x-y) u(t, y)$ is a function in $\mathfrak{H}^{2}$ and $\widetilde{K}_{1}(t)$ is an operator on $\mathfrak{H}$ with kernel $\widetilde{K}_{1}(t, x, y)=u(t, x) w_{N}(x-y) \overline{u(t, y)}$.

In order to extend (2) to the case $\beta>0$, we have to restrict the initial state $\Phi(0)$ in (1) to quasi-free states (namely the states satisfying Wick theorem) with finite kinetic energy. This reduction is again motivated by the rigorous properties of ground states in [32]. Our main result in [41] is

Theorem 1 (Validity of Bogoliubov theory as a norm approximation). Let $\Psi_{N}(t)=$ $e^{-i t H_{N}} \Psi_{N}(0)$ with $\Psi_{N}(0)$ given in (1). We assume

- $u(t)$ satisfies Hartree equation (3) with the (possibly $N$-dependent) initial state $u(0, \cdot)$ satisfying $\|u(0, \cdot)\|_{W^{\ell, 1}\left(\mathbb{R}^{3}\right)} \leq C$ for $\ell$ sufficiently large; 
- $\Phi(t)=\left(\varphi_{n}(t)\right)_{n=0}^{\infty} \in \mathscr{F}_{+}(t)$ satisfies Bogoliubov equation (5) (or equivalently, equation (13) in Section 2) with the (possibly $N$-dependent) initial state $\Phi(0)$ being a quasi-free state in $\mathscr{F}_{+}(0)$ such that for all $\varepsilon>0$,

$$
\langle\Phi(0), \mathscr{N} \Phi(0)\rangle \leq C_{\varepsilon} N^{\varepsilon}, \quad\langle\Phi(0), \mathrm{d} \Gamma(1-\Delta) \Phi(0)\rangle \leq C_{\varepsilon} N^{\beta+\varepsilon} .
$$

Then for all $0 \leq \beta<1 / 2$, all $\varepsilon>0$ and all $t>0$ we have

$$
\left\|\Psi_{N}(t)-\sum_{n=0}^{N} u(t)^{\otimes(N-n)} \otimes_{S} \varphi_{n}(t)\right\|_{\mathfrak{H}^{N}}^{2} \leq C_{\mathcal{E}}(1+t)^{1+\varepsilon} N^{(2 \beta-1+\varepsilon) / 2} .
$$

Convention. We always denote by $C$ (or $C_{\varepsilon}$ ) a general positive constant independent of $N$ and $t\left(C_{\varepsilon}\right.$ may depend on $\left.\varepsilon\right)$.

There are grand canonical analogues of (2) related to the fluctuations around coherent states in Fock space [29, 21, 22, 26, 27, 24, 30]. In particular, our Theorem 1 is comparable to the Fock-space result of Kuz [30]. Thanks to a heuristic argument in [30], the range $0 \leq \beta<1 / 2$ is optimal for the norm approximation (2) to hold.

When $\beta>1 / 2$, to achieve (2) we have to modify the effective equations to take two-body scattering processes into account. This has been done in the Fock space setting by Boccato, Cenatiempo and Schlein [9] and Grillakis and Machedon [25] (see also [5] for a related study). Similar results for $N$-particle dynamics are still open and we hope to be able to come back to this problem in the future.

The proof of Theorem 1 in [41] is built up on the previous works [31] and [40]. The main new ingredient is the following kinetic estimate.

Theorem 2 (Kinetic estimate). Let $\Psi_{N}(0)$ as in Theorem 1. Then for all $0<\beta<$ $1 / 2$, all $\varepsilon>0$ and all $t>0$, we have

$$
\left\langle\Psi_{N}(t), \mathrm{d} \Gamma(Q(t)(1-\Delta) Q(t)) \Psi_{N}(t)\right\rangle \leq C_{\varepsilon}\left(N^{\beta+\varepsilon}+N^{3 \beta-1}\right) .
$$

We can introduce the density matrix $\gamma_{\Psi_{N}(t)}^{(1)}: \mathfrak{H} \rightarrow \mathfrak{H}$ with kernel $\gamma_{\Psi_{N}(t)}^{(1)}(x, y)=$ $\left\langle\Psi_{N}(t), a_{y}^{*} a_{x} \Psi_{N}(t)\right\rangle$ and rewrite (8) as

$$
\operatorname{Tr}\left(\sqrt{1-\Delta} Q(t) \gamma_{\Psi_{N}(t)}^{(1)} Q(t) \sqrt{1-\Delta}\right) \leq C_{\varepsilon}\left(N^{\beta+\varepsilon}+N^{3 \beta-1}\right) .
$$

By the Cauchy-Schwarz inequality, (9) implies that for all $0<\beta<2 / 3$,

$$
\lim _{N \rightarrow \infty} \operatorname{Tr}\left|\sqrt{1-\Delta}\left(N^{-1} \gamma_{\Psi_{N}}^{(1)}-|u(t)\rangle\langle u(t)|\right) \sqrt{1-\Delta}\right|=0
$$

(see Section 3 for more details). In case $\beta=0$, the approximation of the form (10) has been studied in $[38,37,3,39]$. Note that $(10)$ is stronger than the standard definition of the Bose-Einstein condensation

$$
\left.\lim _{N \rightarrow \infty} \operatorname{Tr}\left|N^{-1} \gamma_{\Psi_{N}}^{(1)}-\right| u(t)\right\rangle\langle u(t)||=0
$$


which has been studied by many authors; see $[48,7,20,1,16]$ for some pioneer works (in these works, the convergence (11) was derived using the BBGKY hierarchy, a method that is less quantitative than our approach).

Note that when $\beta=1$ (the Gross-Pitaevskii regime), the strong correlations between particles require a subtle correction: the nonlinear term $w_{N} *|u(t)|^{2}$ in Hartree equation (3) has to be replaced by $8 \pi a|u(t)|^{2}$ with $a$ being the scattering length of $w$. This has been justified rigorously in the context of the Bose-Einstein condensation (11); see [34, 33, 43] for the ground state problem and [18, 17, 8, 45] for the dynamical problem. The norm approximation is completely open.

In the rest, we discuss Hartree and Bogoliubov equations in Section 2, and then go to the proofs of Theorems 2 and Theorem 1 in Sections 3 and 4, respectively.

\section{Effective equations}

We recall the well-posedness of Hartree equation from [24, Prop. 3.3 \& Cor. 3.4].

Lemma 1 (Hartree equation). If $u(0, \cdot) \in H^{2}\left(\mathbb{R}^{3}\right)$, then Hartree equation (3) has a unique global solution $u \in C\left([0, \infty), H^{2}\left(\mathbb{R}^{3}\right)\right) \cap C^{1}\left((0, \infty), L^{2}\left(\mathbb{R}^{3}\right)\right)$. Moreover, if $\|u(0, \cdot)\|_{W^{\ell, 1}\left(\mathbb{R}^{3}\right)} \leq C$ with $\ell$ sufficiently large, then

$$
\|u(t, \cdot)\|_{H^{2}} \leq C, \quad\left\|\partial_{t} u(t, \cdot)\right\|_{L^{2}} \leq C, \quad\|u(t, \cdot)\|_{L^{\infty}}+\left\|\partial_{t} u(t, \cdot)\right\|_{L^{\infty}} \leq C(1+t)^{-3 / 2} .
$$

As in [32, Sec. 2.3], any vector $\Psi \in \mathfrak{H}^{N}$ can be written uniquely as

$$
\Psi=\sum_{n=0}^{N} u(t)^{\otimes(N-n)} \otimes_{s} \varphi_{n}=\sum_{n=0}^{N} \frac{\left(a^{*}(u(t))\right)^{N-n}}{\sqrt{(N-n) !}} \varphi_{n}
$$

with $\varphi_{n} \in \mathfrak{H}_{+}(t)^{n}$. This gives rise to the unitary operator $U_{N}(t): \mathfrak{H}^{N} \rightarrow \mathbb{1}^{\leq N} \mathscr{F}_{+}(t)$

$$
U_{N}(t) \Psi=\varphi_{0} \oplus \varphi_{1} \oplus \cdots \oplus \varphi_{N} .
$$

Here $\mathbb{1}^{\leq N}$ for the projection onto $\mathbb{C} \oplus \mathfrak{H} \oplus \cdots \oplus \mathfrak{H}^{N}$. Some fundamental properties of $U_{N}(t)$ can be found in [32, Proposition 4.2] and [31, Lemma 6].

Next, as in [31], we introduce $\Phi_{N}(t):=U_{N}(t) \Psi_{N}(t)$ and rewrite Schrödinger equation as

$$
i \partial_{t} \Phi_{N}(t)=\widetilde{H}_{N}(t) \Phi_{N}(t), \quad \Phi_{N}(0)=\mathbb{1}^{\leq N} \Phi(0) .
$$

Here $\widetilde{H}_{N}(t)=\mathbb{1} \leq N\left[\mathbb{H}(t)+\frac{1}{2} \sum_{j=0}^{4}\left(R_{j}+R_{j}^{*}\right)\right] \mathbb{1} \leq N$ with

$$
\begin{aligned}
& R_{0}=\mathrm{d} \Gamma\left(Q(t)\left[w_{N} *|u(t)|^{2}+\widetilde{K}_{1}(t)-\mu_{N}(t)\right] Q(t)\right) \frac{1-\mathscr{N}}{N-1}, \\
& R_{1}=-2 \frac{\mathscr{N} \sqrt{N-\mathscr{N}}}{N-1} a\left(Q(t)\left[w_{N} *|u(t)|^{2}\right] u(t)\right),
\end{aligned}
$$




$$
\begin{gathered}
R_{2}=\iint K_{2}(t, x, y) a_{x}^{*} a_{y}^{*} \mathrm{~d} x \mathrm{~d} y\left(\frac{\sqrt{(N-\mathscr{N})(N-\mathscr{N}-1)}}{N-1}-1\right) \\
R_{3}=\frac{\sqrt{N-\mathscr{N}}}{N-1} \iiint \int\left(1 \otimes Q(t) w_{N} Q(t) \otimes Q(t)\right)\left(x, y ; x^{\prime}, y^{\prime}\right) \times \\
\times \overline{u(t, x)} a_{y}^{*} a_{x^{\prime}} a_{y^{\prime}} \mathrm{d} x \mathrm{~d} y \mathrm{~d} x^{\prime} \mathrm{d} y^{\prime}, \\
R_{4}=\frac{1}{2(N-1)} \iiint \int\left(Q(t) \otimes Q(t) w_{N} Q(t) \otimes Q(t)\right)\left(x, y ; x^{\prime}, y^{\prime}\right) \times \\
\times a_{x}^{*} a_{y}^{*} a_{x^{\prime}} a_{y^{\prime}} \mathrm{d} x \mathrm{~d} y \mathrm{~d} x^{\prime} \mathrm{d} y^{\prime} .
\end{gathered}
$$

(In $R_{0}$ and $R_{1}$ we write $w_{N}$ for the function $w_{N}(x)$, while in $R_{3}$ and $R_{4}$ we write $w_{N}$ for the two-body multiplication operator $w_{N}(x-y)$.)

The idea of Bogoliubov approximation is that when $N \rightarrow \infty$ all error terms $R_{j}$ 's are so small that we can ignore them and replace (12) by Bogoliubov equation (5). Some important properties of this equation are collected in the following

Lemma 2 (Bogoliubov equation). (i) If $\Phi(0)$ belongs to the quadratic form domain $\mathscr{Q}(\mathrm{d} \Gamma(1-\Delta))$, then equation (5) has a unique global solution in $\mathscr{Q}(\mathrm{d} \Gamma(1-\Delta))$. Moreover, the pair of density matrices $\left(\gamma_{\Phi(t)}, \alpha_{\Phi(t)}\right)$ is the unique solution to

$$
\left\{\begin{aligned}
i \partial_{t} \gamma & =h \gamma-\gamma h+K_{2} \alpha-\alpha^{*} K_{2}^{*}, \\
i \partial_{t} \alpha & =h \alpha+\alpha h^{\mathrm{T}}+K_{2}+K_{2} \gamma^{\mathrm{T}}+\gamma K_{2}, \\
\gamma(t & =0)=\gamma_{\Phi(0)}, \quad \alpha(t=0)=\alpha_{\Phi(0)} .
\end{aligned}\right.
$$

(ii) If we assume further that $\Phi(0)$ is a quasi-free state in $\mathscr{F}_{+}(0)$, then $\Phi(t)$ is a quasi-free state in $\mathscr{F}_{+}(t)$ for all $t>0$ and

$$
\langle\Phi(t), \mathscr{N} \Phi(t)\rangle \leq C\left(\langle\Phi(0), \mathscr{N} \Phi(0)\rangle^{2}+[\log (2+t)]^{2}\right) .
$$

Recall that $\gamma_{\Phi(t)}: \mathfrak{H} \rightarrow \mathfrak{H}, \alpha_{\Phi(t)}: \overline{\mathfrak{H}} \equiv \mathfrak{H}^{*} \rightarrow \mathfrak{H}$ are operators with kernels $\gamma_{\Phi(t)}(x, y)=\left\langle\Phi(t), a_{y}^{*} a_{x} \Phi(t)\right\rangle, \alpha_{\Phi(t)}(x, y)=\left\langle\Phi(t), a_{x} a_{y} \Phi(t)\right\rangle$ and $K_{2}: \overline{\mathfrak{H}} \equiv \mathfrak{H}^{*} \rightarrow \mathfrak{H}$ is an operator with kernel $K_{2}(t, x, y)$. Note that (13) is similar (but not identical) to the effective equations used in the Fock space setting in $[24,30]$.

Proof. (i) For existence and uniqueness of $\Phi(t)$, we refer to [31, Theorem 7]. To derive (13), we use (5) to compute

$$
\begin{aligned}
& i \partial_{t} \gamma_{\Phi(t)}\left(x^{\prime}, y^{\prime}\right)=i \partial_{t}\left\langle\Phi(t), a_{y^{\prime}}^{*} a_{x} \Phi(t)\right\rangle=\left\langle\Phi(t),\left[a_{y^{\prime}}^{*} a_{x}, \mathbb{H}(t)\right] \Phi(t)\right\rangle \\
& =\iint h(t, x, y)\left(\delta\left(x^{\prime}-x\right) \gamma_{\Phi(t)}\left(y, y^{\prime}\right)-\delta\left(y^{\prime}-y\right) \gamma_{\Phi(t)}\left(x^{\prime}, x\right)\right) \mathrm{d} x \mathrm{~d} y \\
& +\frac{1}{2} \iint k(t, x, y)\left(\delta\left(x^{\prime}-x\right) \alpha_{\Phi(t)}^{*}\left(y, y^{\prime}\right)+\delta\left(x^{\prime}-y\right) \alpha_{\Phi(t)}^{*}\left(y^{\prime}, x\right)\right) \mathrm{d} x \mathrm{~d} y \\
& -\frac{1}{2} \iint k^{*}(t, x, y)\left(\delta\left(y^{\prime}-y\right) \alpha_{\Phi(t)}\left(x, x^{\prime}\right)+\delta\left(y^{\prime}-x\right) \alpha_{\Phi(t)}\left(y, x^{\prime}\right)\right) \mathrm{d} x \mathrm{~d} y
\end{aligned}
$$




$$
=\left(h(t) \gamma_{\Phi(t)}-\gamma_{\Phi(t)} h(t)+K_{2}(t) \alpha_{\Phi(t)}^{*}-\alpha_{\Phi(t)} K_{2}^{*}(t)\right)\left(x^{\prime}, y^{\prime}\right)
$$

This is the first equation in (13). The second equation is proved similarly.

(ii) Now we show that if $\Phi(0)$ is a quasi-free state, then $\Phi(t)$ is a quasi-free state for all $t>0$. We will write $(\gamma, \alpha)=\left(\gamma_{\Phi(t)}, \alpha_{\Phi(t)}\right)$ for short. Let us introduce

$$
X:=\gamma+\gamma^{2}-\alpha \alpha^{*}, \quad Y:=\gamma \alpha-\alpha \gamma^{\mathrm{T}} .
$$

It is a general fact (see, e.g., [40, Lemma 8]) that $\Phi(t)$ is a quasi-free state if and only if $X(t)=0$ and $Y(t)=0$. In particular, we have $X(0)=0$ and $Y(0)=0$ by the assumption on $\Phi(0)$. Using (13) it is straightforward to see that

$$
\begin{aligned}
i \partial_{t} X & =h X-X h+k Y^{*}-Y k^{*}, \\
i \partial_{t} X^{2} & =\left(i \partial_{t} X\right) X+X\left(i \partial_{t} X\right)=h X^{2}-X^{2} h+\left(K_{2} Y^{*}-Y K_{2}^{*}\right) X+X\left(K_{2} Y^{*}-Y K_{2}^{*}\right) .
\end{aligned}
$$

Then we take the trace and use $\operatorname{Tr}\left(h X^{2}-X^{2} h\right)=0\left(h X^{2}\right.$ and $X^{2} h$ may be not trace class but we can introduce a cut-off; see [40] for details). We find that

$$
\|X(t)\|_{\mathrm{HS}}^{2} \leq 4 \int_{0}^{t}\left\|K_{2}(s)\right\| \cdot\|X(s)\|_{\mathrm{HS}} \cdot\|Y(s)\|_{\mathrm{HS}} \mathrm{d} s
$$

We also obtain a similar bound for $\|Y(t)\|_{\mathrm{HS}}$. Then summing these estimates and using the fact that $\left\|K_{2}(t)\right\|$ is bounded uniformly in time, we conclude by Grönwall's inequality that $X(t)=0, Y(t)=0$ for all $t>0$.

A similar argument can be used to the uniqueness of solutions to (13).

To obtain (14), we first estimate $\|\alpha\|_{\mathrm{HS}}^{2}+\|\gamma\|_{\mathrm{HS}}^{2}$ by a Grönwall-type inequality, and then use the identity $\|\alpha\|_{\mathrm{HS}}^{2}=\operatorname{Tr}\left(\gamma+\gamma^{2}\right)$. We refer to [40] for details.

\section{Kinetic bounds}

In this section, we discuss Theorem 2. As mentioned, it is equivalent to (9) and in case $\beta<2 / 3$ it implies (10). Let us explain the implication from (9) to (10) in more details. We will write $P(t)=|u(t)\rangle\langle u(t)|$ for short. We can decompose

$$
\begin{aligned}
N^{-1} \gamma_{\Psi_{N}(t)}^{(1)}-P(t)= & N^{-1} Q(t) \gamma_{\Psi_{N}(t)}^{(1)} Q(t)-N^{-1} \operatorname{Tr}\left(Q(t) \gamma_{\Psi_{N}(t)}^{(1)} Q(t)\right) P(t) \\
& +N^{-1} Q(t) \gamma_{\Psi_{N}(t)}^{(1)} P(t)+N^{-1} P(t) \gamma_{\Psi_{N}(t)}^{(1)} Q(t)
\end{aligned}
$$

and use the triangle inequality of the trace norm to estimate

$$
\begin{aligned}
& \operatorname{Tr}\left|\sqrt{1-\Delta}\left(N^{-1} \gamma_{\Psi_{N}}^{(1)}-|u(t)\rangle\langle u(t)|\right) \sqrt{1-\Delta}\right| \\
& \leq N^{-1} \operatorname{Tr}\left(\sqrt{1-\Delta} Q(t) \gamma_{\Psi_{N}(t)}^{(1)} Q(t) \sqrt{1-\Delta}\right)+N^{-1} \operatorname{Tr}\left(Q(t) \gamma_{\Psi_{N}(t)}^{(1)} Q(t)\right)\|u(t, \cdot)\|_{H^{1}}^{2}
\end{aligned}
$$




$$
+2 N^{-1} \operatorname{Tr}\left|\sqrt{1-\Delta} Q(t) \gamma_{\Psi_{N}(t)}^{(1)} P(t) \sqrt{1-\Delta}\right|
$$

Using the Cauchy-Schwarz inequality (for Schatten norm)

$$
\begin{aligned}
& \operatorname{Tr}\left|(1-\Delta)^{1 / 2} Q(t) \gamma_{\Psi_{N}(t)}^{(1)} P(t)(1-\Delta)^{1 / 2}\right| \\
& \leq\left\|(1-\Delta)^{1 / 2} Q(t)\left(\gamma_{\Psi_{N}(t)}^{(1)}\right)^{1 / 2}\right\|_{\mathrm{HS}} \cdot\left\|\left(\gamma_{\Psi_{N}(t)}^{(1)}\right)^{1 / 2}\right\| \cdot\left\|P(t)(1-\Delta)^{1 / 2}\right\|_{\mathrm{HS}}
\end{aligned}
$$

we deduce from (9) and (15) that for all $\varepsilon>0$,

$$
\operatorname{Tr}\left|\sqrt{1-\Delta}\left(N^{-1} \gamma_{\Psi_{N}}^{(1)}-|u(t)\rangle\langle u(t)|\right) \sqrt{1-\Delta}\right| \leq C_{\varepsilon} N^{a+\varepsilon}
$$

where $a=\max \{\beta-1,(\beta-1) / 2,3 \beta-2,(3 \beta-2) / 2\}$. If $\beta<2 / 3$, then (10) holds.

Now we turn to another version of Theorem 2. From the definition $\Phi_{N}(t)=$ $U_{N}(t) \Psi_{N}(t)$, we can check that $Q(t) \gamma_{\Psi_{N}}^{(1)} Q(t)=\gamma_{\Phi_{N}}^{(1)}$ (e.g. by using [32, Proposition 4.2]). Thus Theorem 2 is equivalent to

Theorem 3 (Kinetic estimate). Let $\Phi_{N}(t)$ be as in (12), with $\Phi(0)$ as in Theorem 1. Then for all $\varepsilon>0$ and all $t>0$, we have

$$
\left\langle\Phi_{N}(t), \mathrm{d} \Gamma(1-\Delta) \Phi_{N}(t)\right\rangle \leq C_{\varepsilon}\left(N^{\beta+\varepsilon}+N^{3 \beta-1+\varepsilon}\right) .
$$

Before proving Theorem 3, let us start with a simpler bound.

Lemma 3 (Bogoliubov kinetic bound). Let $\Phi(t)$ be as in Theorem 1. Then

$$
\langle\Phi(t), \mathrm{d} \Gamma(1-\Delta) \Phi(t)\rangle \leq C_{\varepsilon} N^{\beta+\varepsilon}, \quad \forall t>0
$$

Proof. For a general quadratic Hamiltonian, we have

$$
\mathrm{d} \Gamma(H)+\frac{1}{2} \iint\left(K(x, y) a_{x}^{*} a_{y}^{*}+\overline{K(x, y)} a_{x} a_{y}\right) \mathrm{d} x \mathrm{~d} y \geq-\frac{1}{2} \iint \mid\left(\left.H_{x}^{-1 / 2} K(x, y)\right|^{2} \mathrm{~d} x \mathrm{~d} y .\right.
$$

This bound can be found in our recent joint work with Solovej [42, Lemma 9] (see also [12, Theorem 5.4] for a similar result). Combining this with the Sobolev-type estimate (see [41, Lemma 6])

$$
\left\|\left(1-\Delta_{x}\right)^{-1 / 2} K_{2}(t, \cdot, \cdot)\right\|_{L^{2}}^{2}+\left\|\left(1-\Delta_{x}\right)^{-1 / 2} \partial_{t} K_{2}(t, \cdot, \cdot)\right\|_{L^{2}}^{2} \leq C_{\varepsilon}(1+t)^{-3} N^{\beta+\varepsilon}
$$

we obtain the quadratic form inequalities (see [41, Lemma 7])

$$
\begin{array}{r} 
\pm(\mathbb{H}(t)+\mathrm{d} \Gamma(\Delta)) \leq \eta \mathrm{d} \Gamma(1-\Delta)+\frac{C_{\varepsilon}\left(\mathscr{N}+N^{\beta+\varepsilon}\right)}{\eta(1+t)^{3}} \\
\pm \partial_{t} \mathbb{H}(t) \leq \eta \mathrm{d} \Gamma(1-\Delta)+\frac{C_{\varepsilon}\left(\mathscr{N}+N^{\beta+\varepsilon}\right)}{\eta(1+t)^{3}} \\
\pm i[\mathbb{H}(t), \mathscr{N}] \leq \eta \mathrm{d} \Gamma(1-\Delta)+\frac{C_{\varepsilon}\left(\mathscr{N}+N^{\beta+\varepsilon}\right)}{\eta(1+t)^{3}}
\end{array}
$$


for all $\eta>0$. On the other hand, from Bogoliubov equation (5), we have

$$
\langle\Phi(t), \mathbb{H}(t) \Phi(t)\rangle-\langle\Phi(0), \mathbb{H}(0) \Phi(0)\rangle=\int_{0}^{t}\left\langle\Phi(s), \partial_{s} \mathbb{H}(s) \Phi(s)\right\rangle \mathrm{d} s .
$$

Using (18) with $\eta=1 / 2$ we have $\langle\Phi(0), \mathbb{H}(0) \Phi(0)\rangle \leq C_{\varepsilon} N^{\beta+\varepsilon}$ and

$$
\langle\Phi(t), \mathbb{H}(t) \Phi(t)\rangle \geq \frac{1}{2}\langle\Phi(t), \mathrm{d} \Gamma(1-\Delta) \Phi(t)\rangle-C_{\varepsilon}\left(\langle\Phi(t), \mathscr{N} \Phi(t)\rangle+N^{\beta+\varepsilon}\right)
$$

Using (19) with $\eta=(1+t)^{-3 / 2}$ we get

$$
\left\langle\Phi(t), \partial_{t} \mathbb{H}(t) \Phi(t)\right\rangle \leq C_{\varepsilon}(1+t)^{-3 / 2}\left(\langle\Phi(t), \mathrm{d} \Gamma(1-\Delta) \Phi(t)\rangle+N^{\beta+\varepsilon}\right) .
$$

Thus (21) implies that

$$
\begin{aligned}
\langle\Phi(t), \mathrm{d} \Gamma(1-\Delta) \Phi(t)\rangle \leq C_{\varepsilon} \int_{0}^{t}(1+s)^{-3 / 2}\langle\Phi(s), \mathrm{d} \Gamma(1-\Delta) \Phi(s)\rangle \mathrm{d} s \\
+C_{\varepsilon}\left(\langle\Phi(t), \mathscr{N} \Phi(t)\rangle+N^{\beta+\varepsilon}\right)
\end{aligned}
$$

Similarly, we can estimate $\partial_{t}\langle\Phi(t), \mathscr{N} \Phi(t)\rangle$ by using Bogoliubov equation (5) and (20) with $\eta=(1+t)^{-3 / 2}$. Then we integrate the resulting bound and obtain

$$
\langle\Phi(t), \mathscr{N} \Phi(t)\rangle \leq C_{\varepsilon} \int_{0}^{t}(1+s)^{-3 / 2}\langle\Phi(s), \mathrm{d} \Gamma(1-\Delta) \Phi(s)\rangle \mathrm{d} s+C_{\varepsilon} N^{\beta+\varepsilon} .
$$

Inserting the latter inequality into the right side of (22) we obtain

$$
\langle\Phi(t), \mathrm{d} \Gamma(1-\Delta) \Phi(t)\rangle \leq C_{\varepsilon}(1+s)^{-3 / 2} \int_{0}^{t}\langle\Phi(s), \mathrm{d} \Gamma(1-\Delta) \Phi(s)\rangle \mathrm{d} s+C_{\varepsilon} N^{\beta+\varepsilon} .
$$

The desired result then follows from a Gronwall-type inequality.

The proof of Theorem 3 is based on a similar argument. We will use the following estimates on the error terms $R_{j}$ 's in (12) (see [41, Lemmas 9, 11]).

Lemma 4 (Control of error terms). Let $R_{j}$ 's be as in (12). Then we have the quadratic form estimates on $\mathbb{1} \leq N \mathscr{F}_{+}(t)$ :

$$
\begin{gathered}
\pm\left(R_{j}+R_{j}^{*}\right) \leq \eta\left(R_{4}+\frac{\mathscr{N}^{2}}{N}\right)+\frac{C(1+\mathscr{N})}{\eta(1+t)^{3}}, \quad \forall \eta>0, \forall j=0,1,2,3, \\
0 \leq R_{4} \leq C N^{3 \beta-1} \mathscr{N}^{2}, \quad R_{4} \leq C N^{\beta-1} \mathrm{~d} \Gamma(-\Delta) \mathscr{N} \\
\pm \partial_{t}\left(R_{j}+R_{j}^{*}\right) \leq \eta\left(R_{4}+\frac{\mathscr{N}^{2}}{N}\right)+\frac{C(1+\mathscr{N})}{\eta(1+t)^{3}}, \forall j=0,1,2,3,4, \\
\pm i\left[\left(R_{j}+R_{j}^{*}\right), \mathscr{N}\right] \leq \eta\left(R_{4}+\frac{\mathscr{N}^{2}}{N}\right)+\frac{C(1+\mathscr{N})}{\eta(1+t)^{3}}, \forall j=0,1,2,3,4 .
\end{gathered}
$$


Now we are ready to provide

Proof (of Theorem 3). From (12) we have

$$
\left\langle\Phi_{N}(t), \widetilde{H}_{N}(t) \Phi_{N}(t)\right\rangle-\left\langle\Phi_{N}(0), \widetilde{H}_{N}(0) \Phi_{N}(0)\right\rangle=\int_{0}^{t}\left\langle\Phi_{N}(s), \partial_{s} \widetilde{H}_{N}(s) \Phi_{N}(s)\right\rangle \mathrm{d} s .
$$

Using (18) and Lemma 4, we can estimate

$$
\begin{gathered}
\left\langle\Phi_{N}(t), \widetilde{H}_{N}(t) \Phi_{N}(t)\right\rangle \geq \frac{1}{2}\left\langle\Phi_{N}(t),\left(\mathrm{d} \Gamma(1-\Delta)+R_{4}\right) \Phi_{N}(t)\right\rangle \\
-C_{\varepsilon}\left(N^{\beta+\varepsilon}+\left\langle\Phi_{N}(t), \mathscr{N} \Phi_{N}(t)\right\rangle\right), \\
\left\langle\Phi_{N}(0), \widetilde{H}_{N}(0) \Phi_{N}(0)\right\rangle \leq C_{\varepsilon}\left(N^{\beta+\varepsilon}+N^{3 \beta-1+\varepsilon}\right) .
\end{gathered}
$$

Here in the last inequality, we have used $R_{4} \leq C N^{3 \beta-1} \mathscr{N}^{2}$ (see Lemma 4) and a well-known moment estimate for every quasi-free state $\Phi$ :

$$
\left\langle\Phi,(1+\mathscr{N})^{s} \Phi\right\rangle \leq C_{s}\langle\Phi,(1+\mathscr{N}) \Phi\rangle^{s}
$$

where the constant $C_{s}$ depends only on $s \in \mathbb{N}$ (see e.g. [40, Lemma 5]). Moreover, from (19) and Lemma 4 we obtain

$\left\langle\Phi_{N}(t), \partial_{t} \widetilde{H}_{N}(t) \Phi_{N}(t)\right\rangle \leq C_{\varepsilon}(1+t)^{-3 / 2}\left(\left\langle\Phi_{N}(t),\left(\mathrm{d} \Gamma(1-\Delta)+R_{4}\right) \Phi_{N}(t)\right\rangle+N^{\beta+\varepsilon}\right)$.

Thus (23) implies that

$$
\begin{aligned}
\left\langle\Phi_{N}(t),(\mathrm{d} \Gamma(1-\Delta)\right. & \left.\left.+R_{4}\right) \Phi_{N}(t)\right\rangle \leq C_{\varepsilon} \int_{0}^{t} \frac{\left\langle\Phi_{N}(s),\left(\mathrm{d} \Gamma(1-\Delta)+R_{4}\right) \Phi_{N}(s)\right\rangle}{(1+s)^{3 / 2}} \mathrm{~d} s \\
& +C_{\varepsilon}\left(N^{\beta+\varepsilon}+N^{3 \beta-1+\varepsilon}+\left\langle\Phi_{N}(t), \mathscr{N} \Phi_{N}(t)\right\rangle\right)
\end{aligned}
$$

Next, we estimate $\partial_{t}\left\langle\Phi_{N}(t), \mathscr{N} \Phi_{N}(t)\right\rangle$ by using (12), (20) and the last inequality in Lemma 4. Then we integrate the resulting bound to get

$$
\langle\Phi(t), \mathscr{N} \Phi(t)\rangle \leq C_{\varepsilon} \int_{0}^{t}(1+s)^{-3 / 2}\left\langle\Phi_{N}(s),\left(\mathrm{d} \Gamma(1-\Delta)+R_{4}\right) \Phi_{N}(s)\right\rangle \mathrm{d} s+C_{\varepsilon} N^{\beta+\varepsilon}
$$

Substituting the latter estimate into (25), we find that

$$
\begin{aligned}
& \left\langle\Phi_{N}(t),\left(\mathrm{d} \Gamma(1-\Delta)+R_{4}\right) \Phi_{N}(t)\right\rangle \\
& \leq C_{\varepsilon} \int_{0}^{t} \frac{\left\langle\Phi_{N}(s),\left(\mathrm{d} \Gamma(1-\Delta)+R_{4}\right) \Phi_{N}(s)\right\rangle}{(1+s)^{3 / 2}} \mathrm{~d} s+C_{\varepsilon}\left(N^{\beta+\varepsilon}+N^{3 \beta-1+\varepsilon}\right) .
\end{aligned}
$$

By a Gronwall-type inequality, we conclude that

$$
\left\langle\Phi_{N}(t),\left(\mathrm{d} \Gamma(1-\Delta)+R_{4}\right) \Phi_{N}(t)\right\rangle \leq C_{\varepsilon}\left(N^{\beta+\varepsilon}+N^{3 \beta-1+\varepsilon}\right) .
$$


Since $R_{4} \geq 0$, the desired kinetic estimate follows.

\section{Norm approximation}

Proof (of Theorem 1). Step 1. The desired estimate (7) is

$$
\left\|\Psi_{N}(t)-U_{N}(t)^{*} \mathbb{1}^{\leq N} \Phi(t)\right\|_{\mathfrak{H}^{N}}^{2} \leq C_{\varepsilon}(1+t)^{1+\varepsilon} N^{(2 \beta+\varepsilon-1) / 2}, \quad \forall \varepsilon>0 .
$$

Since $\Phi(t)=U_{N}(t) \Psi_{N}(t)$ and $U_{N}(t): \mathfrak{H}^{N} \rightarrow \mathbb{1}^{\leq N} \mathscr{F}_{+}(t)$ is a unitary operator,

$$
\left\|\Psi_{N}(t)-U_{N}(t)^{*} \mathbb{1}^{\leq N} \Phi(t)\right\|_{\mathfrak{H}^{N}}=\left\|U_{N}(t) \Psi_{N}(t)-\mathbb{1}^{\leq N} \Phi(t)\right\| \leq\left\|\Phi_{N}(t)-\Phi(t)\right\| .
$$

It remains to bound $\left\|\Phi_{N}(t)-\Phi(t)\right\|$. Using equations (5) and (12), we can write

$$
\begin{aligned}
\partial_{t}\left\|\Phi_{N}(t)-\Phi(t)\right\|^{2} & =2 \Re\left\langle i \Phi_{N}(t),\left(\widetilde{H}_{N}(t)-\mathbb{H}(t)\right) \Phi(t)\right\rangle \\
& =\sum_{j=0}^{4} \Re\left\langle i \Phi_{N}(t),\left(R_{j}+R_{j}^{*}\right) \mathbb{1}^{\leq N} \Phi(t)\right\rangle-2 \Re\left\langle i \Phi_{N}(t), \mathbb{H} \mathbb{1}^{>N} \Phi(t)\right\rangle
\end{aligned}
$$

where $\mathbb{1}^{>N}:=\mathbb{1}-\mathbb{1}^{\leq N}$. Next, we will estimate the right side of (26).

Step 2. To bound the last term of (26), we use $\Phi_{N}(t) \in \mathbb{1} \leq N \mathscr{F}_{+}(t)$ to write

$$
\left\langle\Phi_{N}(t), \mathbb{H} \mathbb{1}^{>N} \Phi(t)\right\rangle=\left\langle\Phi_{N}(t),(\mathbb{H}-\mathrm{d} \Gamma(h)) \mathbb{1}^{>N} \Phi(t)\right\rangle .
$$

As in the proof of Lemma 3, we can show that

$$
\pm(\mathbb{H}-\mathrm{d} \Gamma(h)) \leq C\left(\mathscr{N}+N^{3 \beta}\right) .
$$

It is a general fact that if $\pm B \leq A$ as quadratic forms, then we have the CauchySchwarz type inequality $|\langle f, B g\rangle| \leq 3\langle f, A f\rangle^{1 / 2}\langle g, A g\rangle^{1 / 2}$. Consequently,

$$
\begin{aligned}
& \left|\left\langle\Phi_{N}(t),(\mathbb{H}-\mathrm{d} \Gamma(h)) \mathbb{1}^{>N} \Phi(t)\right\rangle\right| \\
& \leq 3\left\langle\Phi_{N}(t),\left(\mathscr{N}+N^{3 \beta}\right) \Phi_{N}(t)\right\rangle^{1 / 2}\left\langle\mathbb{1}^{>N} \Phi(t),\left(\mathscr{N}+N^{3 \beta}\right) \mathbb{1}^{>N} \Phi(t)\right\rangle^{1 / 2} \\
& \left.\leq 3\left(N+N^{3 \beta}\right)^{1 / 2}\right\rangle^{1 / 2}\left\langle\mathbb{1}^{>N} \Phi(t),\left(\mathscr{N}+N^{3 \beta}\right) \mathscr{N}^{s} N^{-s} \mathbb{1}^{>N} \Phi(t)\right\rangle^{1 / 2}
\end{aligned}
$$

for all $s \geq 1$. The term $\left\langle\Phi(t), \mathscr{N}^{s} \Phi(t)\right\rangle$ can be bounded by (24) and the bound on $\langle\Phi(t), \mathscr{N} \Phi(t)\rangle$ in Lemma 2. We can choose $s$ large enough (but fixed) and obtain

$$
\left|\left\langle\Phi_{N}(t), \mathbb{H} \mathbb{1}^{>N} \Phi(t)\right\rangle\right| \leq C_{\varepsilon}(1+t)^{\varepsilon} N^{-1} .
$$

Step 3. To control the first term on the right side of (26), we have to introduce a cut-off on the number of particles. Since there are at most 2 creation or annihilation operators in the expressions of $R_{j}$ 's, we can write 


$$
\begin{aligned}
\left\langle\Phi_{N}(t),\left(R_{j}+R_{j}^{*}\right) \mathbb{1}^{\leq N} \Phi(t)\right\rangle= & \left\langle\mathbb{1}^{\leq M} \Phi_{N}(t),\left(R_{j}+R_{j}^{*}\right) \mathbb{1}^{\leq M+2} \Phi(t)\right\rangle \\
& +\left\langle\mathbb{1}^{>M} \Phi_{N}(t),\left(R_{j}+R_{j}^{*}\right) \mathbb{1}^{\leq N} \mathbb{1}^{>M-2} \Phi(t)\right\rangle
\end{aligned}
$$

for all $4<M<N-2$. Then we estimate each term on the right side by Lemma 4 and the Cauchy-Schwarz type inequality as in Step 2. We obtain

$$
\left|\left\langle\Phi_{N}(t),\left(R_{j}+R_{j}^{*}\right) \mathbb{1}^{\leq N} \Phi(t)\right\rangle\right| \leq C\left(E_{1}+E_{2}\right)
$$

where

$$
\begin{aligned}
E_{1}=\inf _{\eta>0} & \left\langle\mathbb{1}^{\leq M} \Phi_{N}(t),\left((1+\eta) R_{4}+\eta \frac{\mathscr{N}^{2}}{N}+\frac{1+\mathscr{N}}{\eta(1+t)^{3}}\right) \mathbb{1}^{\leq M} \Phi_{N}(t)\right\rangle^{1 / 2} \\
& \times\left\langle\mathbb{1}^{\leq M+2} \Phi(t),\left((1+\eta) R_{4}+\eta \frac{\mathscr{N}^{2}}{N}+\frac{1+\mathscr{N}}{\eta(1+t)^{3}}\right) \mathbb{1}^{\leq M+2} \Phi(t)\right\rangle^{1 / 2}, \\
E_{2}=\inf _{\eta>0} & \left\langle\mathbb{1}^{>M} \Phi_{N}(t),\left((1+\eta) R_{4}+\eta \frac{\mathscr{N}^{2}}{N}+\frac{1+\mathscr{N}}{\eta(1+t)^{3}}\right) \mathbb{1}^{>M} \Phi_{N}(t)\right\rangle^{1 / 2} \\
& \times\left\langle\mathbb{1}^{>M-2} \Phi(t),\left((1+\eta) R_{4}+\eta \frac{\mathscr{N}^{2}}{N}+\frac{1+\mathscr{N}}{\eta(1+t)^{3}}\right) \mathbb{1}^{>M-2} \Phi(t)\right\rangle^{1 / 2} .
\end{aligned}
$$

To bound $E_{1}$, we use

$$
\mathbb{1}^{\leq M} R_{4} \leq C N^{\beta-1} \mathbb{1} \leq M \mathscr{N} \mathrm{d} \Gamma(-\Delta) \leq C N^{\beta-1} M \mathrm{~d} \Gamma(-\Delta)
$$

(see Lemma 4) together with the kinetic estimate in Theorem 3, and then optimize over $\eta>0$. We get

$$
E_{1} \leq C_{\varepsilon}\left(M N^{(2 \beta+\varepsilon-1) / 2}+M^{3 / 2} N^{-1 / 2}\right)
$$

(The error term $N^{3 \beta-1+\varepsilon}$ in Theorem 3 is absorbed by $N^{\beta+\varepsilon}$ when $\beta<1 / 2$.)

The bound on $E_{2}$ is obtained using the argument in Step 2 and reads

$$
E_{2} \leq C_{\varepsilon, s} N^{3 \beta+1} M^{1-s / 2} N^{s \varepsilon}[\log (2+t)]^{s} .
$$

In summary, from (28) it follows that

$$
\begin{aligned}
\left|\left\langle\Phi_{N}(t),\left(R_{j}+R_{j}^{*}\right) \mathbb{1}^{\leq N} \Phi(t)\right\rangle\right| & \leq C_{\varepsilon}\left(M N^{(2 \beta+\varepsilon-1) / 2}+M^{3 / 2} N^{-1 / 2}\right) \\
& +C_{\varepsilon, s} N^{3 \beta+1} M^{1-s / 2} N^{s \varepsilon}[\log (2+t)]^{s}
\end{aligned}
$$

for all $4<M<N-2$ and $s \geq 2$. We can choose $M=N^{3 \varepsilon}$ and $s=s(\varepsilon)$ sufficiently large (e.g. $s \geq 6(1+\beta+\varepsilon) / \varepsilon$ ) to obtain

$$
\left|\left\langle\Phi_{N}(t),\left(R_{j}+R_{j}^{*}\right) \mathbb{1}^{\leq N} \Phi(t)\right\rangle\right| \leq C_{\varepsilon}\left(N^{(2 \beta+9 \varepsilon-1) / 2}+N^{-1}(1+t)^{\varepsilon}\right) .
$$


Step 4. Inserting (27) and (29) into (26), we find that

$$
\partial_{t}\left\|\Phi_{N}(t)-\Phi(t)\right\|^{2} \leq C_{\varepsilon}\left(N^{(2 \beta+9 \varepsilon-1) / 2}+N^{-1}(1+t)^{\varepsilon}\right) .
$$

Integrating over $t$ and using

$$
\left\|\Phi_{N}(0)-\Phi(0)\right\|^{2}=\left\langle\Phi(0), \mathbb{1}^{>N} \Phi(0)\right\rangle \leq N^{-1}\langle\Phi(0), \mathscr{N} \Phi(0)\rangle \leq C_{\varepsilon} N^{\varepsilon-1} .
$$

we obtain

$$
\left\|\Phi_{N}(t)-\Phi(t)\right\|^{2} \leq C_{\varepsilon}(1+t)^{1+\varepsilon} N^{(2 \beta+9 \varepsilon-1) / 2}
$$

for all $\varepsilon>0$. This leads to the desired estimate (7), as explained in Step 1 .

\section{References}

1. R. Adami, F. Golse, And A. Teta, Rigorous Derivation of the Cubic NLS in Dimension One, J. Stat. Phys., 127 (6) (2007), pp. 1193-1220.

2. Z. Ammari AND F. NiER, Mean field limit for bosons and infinite dimensional phase-space analysis, Ann. Henri Poincaré, 9 (2008), pp. 1503-1574.

3. I. AnAPOLITANOS AND M. HotT, A simple proof of convergence to the Hartree dynamics in Sobolev trace norms, e-print arxiv:1608.01192 (2016)

4. M.H. Anderson, J.R. Ensher, M.R. Matthews, C.E. Wieman, E.A. Cornell, Observation of Bose-Einstein Condensation in a Dilute Atomic Vapor, Science 269 (1995), pp. 198-201.

5. V. Bach, S. Breteaux, T. Chen, J. Fröhlich and I.M. Sigal, The time-dependent Hartree-Fock-Bogoliubov equations for Bosons, e-print arXiv:1602.05171 (2015).

6. J. BARdeEn, L. N. Cooper, And J. R. SchriefFer, Theory of Superconductivity, Phys. Rev. 108 (1957), p. 1175.

7. C. BARdos, F. Golse, And N.J. MAUSER, Weak coupling limit of the N-particle Schrödinger equation, Methods Appl. Anal., 7(2) (2000), p. 275-293.

8. N. Benedikter, G. De Oliveira, And B. Schlein, Quantitative Derivation of the GrossPitaevskii Equation, Comm. Pure App. Math., 68 (8) (2015), pp. 1399-1482.

9. C. Boccato, S. CEnATIEMPo, AND B. SChlein, Quantum many-body fluctuations around nonlinear Schrödinger dynamics, Ann. Henri Poincaré (2016), doi:10.1007/s00023-016-05136 , available online

10. N. Bogoliubov, On the theory of superfluidity, J. Phys. (USSR) 11 (1947), p. 23.

11. S.N. Bose, Plancks Gesetz und Lichtquantenhypothese, Zeitschrift für Physik 26 (1924), pp. $178-181$

12. L. BRUNEAU AND J. DEREZIŃSKI, Bogoliubov hamiltonians and one-parameter groups of Bogoliubov transformations, J. Math. Phys., 48 (2007), p. 022101.

13. K.B. Davis, M.-O. Mewes, M.R. Andrews, N.J. van Druten, D.S. Durfee, D.M. Kurn, W. Ketterle, Bose-Einstein condensation in a gas of sodium atoms, Phys. Rev. Lett. 75 (1995), pp. 3969-3973.

14. J. DEREZIŃSKI AND M. NAPIÓRKOWSKI, Excitation spectrum of interacting bosons in the mean-field infinite-volume limit, Annales Henri Poincaré, 15 (2014), pp. 2409-2439. Erratum: Annales Henri Poincaré 16 (2015), pp. 1709-1711.

15. A. Einstein, Quantentheorie des einatomigen idealen Gases, Sitzungsberichte der Preussischen Akademie der Wissenschaften 1 (1925), pp. 3. 
16. L. ERdös, B. SChleIn, AND H.-T. YAU, Derivation of the cubic non-linear Schrödinger equation from quantum dynamics of many-body systems, Invent. Math., 167 (2007), pp. 515614.

17. - Rigorous derivation of the Gross-Pitaevskii equation with a large interaction potential, J. Amer. Math. Soc., 22 (2009), pp. 1099-1156.

18. - Derivation of the Gross-Pitaevskii equation for the dynamics of Bose-Einstein condensate, Ann. of Math. (2), 172 (2010), pp. 291-370.

19. — Ground-state energy of a low-density Bose gas: A second-order upper bound, Phys. Rev. A, 78 (2008), p. 053627.

20. L. ERDÖS AND H.-T. YAU, Derivation of the nonlinear Schrödinger equation from a many body Coulomb system, Adv. Theor. Math. Phys., 5 (2001), pp. 1169-1205.

21. J. GinibRe AND G. Velo, The classical field limit of scattering theory for nonrelativistic many-boson systems. I, Commun. Math. Phys., 66 (1979), pp. 37-76.

22. - The classical field limit of scattering theory for nonrelativistic many-boson systems. II, Commun. Math. Phys., 68 (1979), pp. 45-68.

23. P. GRECH AND R. SEIRINGER, The excitation spectrum for weakly interacting bosons in a trap, Comm. Math. Phys., 322 (2013), pp. 559-591.

24. M. GRillakis AND M. Machedon, Pair excitations and the mean field approximation of interacting Bosons, I, Commun. Math. Phys., 324 (2013), pp. 601-636.

25. M. GRILla KIS AND M. MaChedon, Pair excitations and the mean field approximation of interacting Bosons, II, e-print arXiv: 1509.05911 (2015)

26. M. G. Grillakis, M. Machedon, And D. Margetis, Second-order corrections to mean field evolution of weakly interacting bosons. I, Commun. Math. Phys., 294 (2010), pp. 273 301.

27. - Second-order corrections to mean field evolution of weakly interacting bosons. II, Adv. Math., 228 (2011), pp. 1788-1815.

28. A. GiUliani AND R. SEIRINGer, The ground state energy of the weakly interacting Bose gas at high density, J. Stat. Phys., 135 (2009), pp. 915-934.

29. K. HePP, The classical limit for quantum mechanical correlation functions, Comm. Math. Phys., 35 (1974), pp. 265-277.

30. E. KuZ, Exact Evolution versus Mean Field with Second-order correction for Bosons Interacting via Short-range Two-body Potential, e-print arXiv:1511.00487 (2015)

31. M. Lewin, P. T. NAM, AND B. SChleIn, Fluctuations around Hartree states in the meanfield regime, Amer. J. Math., 137 (2015), pp. 1613-1650

32. M. Lewin, P. T. NAm, S. Serfaty, AND J. P. SoloveJ, Bogoliubov spectrum of interacting Bose gases, Comm. Pure Appl. Math., 68 (2015), pp. 413-471.

33. E. H. Lieb AND R. SEIRINGer, Derivation of the Gross-Pitaevskii equation for rotating Bose gases, Commun. Math. Phys., 264 (2006), pp. 505-537.

34. E. H. LIEB, R. SEIRINGER, AND J. YNGVASON, Bosons in a trap: A rigorous derivation of the Gross-Pitaevskii energy functional, Phys. Rev. A, 61 (2000), p. 043602.

35. E. H. Lieb AND J. P. SOlOVEJ, Ground state energy of the one-component charged Bose gas, Commun. Math. Phys., 217 (2001), pp. 127-163.

36. E. H. Lieb AND J. P. Solovej, Ground state energy of the two-component charged Bose gas., Commun. Math. Phys., 252 (2004), pp. 485-534.

37. J. LÜHrmann, Mean-field quantum dynamics with magnetic fields. J. Math. Phys. 53 (2012), p. 022105

38. A. Michelangeli And B. Schlein, Dynamical collapse of boson stars, Commun. Math. Phys. 311 (2012), pp. 645-687.

39. D. Mitrouskas, S. Petrat AND P. Pickl, Bogoliubov corrections and trace norm convergence for the Hartree dynamics, e-print arXiv:1609.06264 (2016)

40. P. T. NAM AND M. NAPIÓRKOWSKI, Bogoliubov correction to the mean-field dynamics of interacting bosons, Adv. Theor. Math. Phys., to appear, e-print arXiv:1509.04631 (2015).

41. P. T. NAM AND M. NAPIÓRKOWSKI, A note on the validity Bogoliubov correction to the mean-field dynamics, J. Math. Pures Appl., to appear, e-print arXiv:1604.05240 (2016). 
42. P. T. NAm, M. Napiórkows Ki AND J. P. Solovej, Diagonalization of bosonic quadratic Hamiltonians by Bogoliubov transformations, J. Funct. Anal., 270 (11) (2016), pp. 4340-4368

43. P.T. NAm, N. Rougerie And R. SEIRINGER, Ground states of large bosonic systems: The Gross-Pitaevskii limit revisited, Anal. \& PDE., 9 (2) (2016), pp. 459-485.

44. P. NAM AND R. SEIRINGER, Collective excitations of Bose gases in the mean-field regime, Arch. Rational Mech. Anal., 215 (2015), pp. 381-417.

45. P. PICKL, Derivation of the time dependent Gross Pitaevskii equation with external fields, Rev. Math. Phys., 27 (2015), p. 1550003.

46. I. RoDNIANSKI AND B. SCHLEIN, Quantum fluctuations and rate of convergence towards mean field dynamics, Commun. Math. Phys., 291 (2009), pp. 31-61.

47. R. SeIRINGER, The excitation spectrum for weakly interacting bosons, Commun. Math. Phys., 306 (2011), pp. 565-578.

48. H. Spohn, Kinetic equations from Hamiltonian dynamics: Markovian limits, Rev. Modern Phys., 52 (1980), pp. 569-615.

49. J. P. SoloveJ, Upper bounds to the ground state energies of the one- and two-component charged Bose gases, Commun. Math. Phys., 266 (2006), pp. 797-818.

50. H.-T. YAU AND J. YIN, The second order upper bound for the ground energy of a Bose gas, J. Stat. Phys., 136 (2009), pp. 453-503. 